\title{
Autophagy and the Kidney: Implications for Ischemia-Reperfusion Injury and Therapy
}

\author{
Jean-Paul Decuypere, PhD, ${ }^{1,2}$ Laurens J. Ceulemans, $M D,{ }^{1,2}$ Patrizia Agostinis, $P h D,{ }^{3}$ \\ Diethard Monbaliu, MD, PhD, ${ }^{1,2}$ Maarten Naesens, MD, PhD, ${ }^{4,5}$ \\ Jacques Pirenne, MD, PhD, ${ }^{1,2}$ and Ina Jochmans, $M D, P h D^{1,2}$
}

\begin{abstract}
Autophagy, an evolutionary conserved intracellular lysosome-dependent catabolic process, is an important mechanism for cellular homeostasis and survival during pathologic stress conditions in the kidney, such as ischemia-reperfusion injury (IRI). However, stimulation of autophagy has been described to both improve and exacerbate IRI in the kidney. We summarize the current understanding of autophagy in renal IRI and discuss possible reasons for these contradictory findings. Furthermore, we hypothesize that autophagy plays a dual role in renal IRI, having both protective and detrimental properties, depending on the duration of the ischemic period and the phase of the IRI process. Finally, we discuss the influence of currently used diuretics and immunosuppressive drugs on autophagy, underscoring the need to clarify the puzzling role of autophagy in renal IRI.

Am J Kidney Dis. 66(4):699-709. (c) 2015 by the National Kidney Foundation, Inc.
\end{abstract}

INDEX WORDS: Autophagy; macroautophagy; cell death; ischemia-reperfusion injury (IRI); renal IRI; acute kidney injury (AKI); stress response; animal model; kidney transplantation; delayed graft function; immunosuppression; review.

\section{BACKGROUND}

Renal ischemia-reperfusion injury (IRI) contributes to acute kidney injury (AKI) and delayed graft function after kidney transplantation. Although renal IRI is a major cause of morbidity and mortality, there are currently no effective treatments. ${ }^{1}$ Because kidney cells rely heavily on autophagy, an evolutionarily conserved intracellular degradation pathway, for their homeostasis and survival during IRI, stimulation of autophagy harbors therapeutic potential. ${ }^{2-6}$ By recycling damaged and toxic cytoplasmic material into new cellular building blocks, autophagy supports antistress responses and energy maintenance. Therefore, it seems logical to reinforce this natural protective mechanism to diminish IRI. ${ }^{7}$ However, paradoxically, autophagy also promotes renal IRI (Table 1). - $6,8-17^{-17}$ Consequently, the exact role of autophagy in renal IRI needs to be clarified to determine whether autophagy stimulation or inhibition best attenuates IRI. ${ }^{18}$ This is particularly important because many drugs applied in the context of AKI (diuretics) or kidney transplantation (immunosuppressants) influence autophagy and therefore affect renal IRI. Unfortunately, in vivo autophagy research is challenging: the dynamic nature of the process, the crosstalk with apoptosis and necrosis, and the lack of clear-cut markers and specific modulators complicate interpretation of results despite published autophagy research guidelines. ${ }^{19}$

This review introduces autophagy in renal IRI to the clinician and explores the reasons for contradictory findings in the literature. It highlights the difficulties surrounding autophagy research and introduces a new hypothesis of a dual role for autophagy in renal IRI (both protective and detrimental), depending on the experimental context.

\section{CASE VIGNETTE}

A 59-year-old man (blood group, O negative; 0\% panel-reactive antibodies) with end-stage renal disease secondary to type 1 diabetes received a kidney transplant from a 53-year-old man (blood group, O positive; crossmatch, negative). The kidney was donated after cardiocirculatory arrest with a donor warm ischemia time of 26 minutes, hypothermic machine preservation (18.5 hours), and cold ischemia time of 21.5 hours. Initial treatment consisted of tacrolimus, mycophenolate mofetil (MMF), and steroids. Delayed graft function occurred. Indication kidney biopsy showed acute cellular rejection grade $2 \mathrm{a}$, acute humoral rejection type 2 , and mild acute tubular necrosis. The patient was treated successfully with highdose steroids. A protocol biopsy 3 months later revealed chronic allograft nephropathy (grade $1 \mathrm{~b}$ ), which remained stable for the following 3 years. At last follow-up, the patient was well with stable kidney function (serum creatinine of $1.54 \mathrm{mg} / \mathrm{dL}$, corresponding to

From the ${ }^{1}$ Department of Microbiology and Immunology, Laboratory of Abdominal Transplantation, KU Leuven, University of Leuven; ${ }^{2}$ Department of Abdominal Transplant Surgery, University Hospitals Leuven; ${ }^{3}$ Department of Cellular and Molecular Medicine, Laboratory of Cell Death Research and Therapy, and ${ }^{4}$ Department of Microbiology and Immunology, Laboratory of Nephrology, KU Leuven, University of Leuven; and ${ }^{5}$ Department of Nephrology, University Hospitals Leuven, Leuven, Belgium.

Received August 25, 2014. Accepted in revised form May 21, 2015. Originally published online July 11, 2015.

Address correspondence to Jean-Paul Decuypere, PhD, Laboratory of Abdominal Transplantation, Department of Microbiology and Immunology, Faculty of Medicine, KU Leuven, Campus Gasthuisberg, Herestraat 49, Box 7003, B-3000, Leuven, Belgium.

E-mail: jeanpaul.decuypere@med.kuleuven.be

(C) 2015 by the National Kidney Foundation, Inc.

0272-6386

http://dx.doi.org/10.1053/j.ajkd.2015.05.021 
Table 1. Overview of Different Studies of Autophagy in Renal IRI

\begin{tabular}{|c|c|c|c|c|c|c|}
\hline Study & Model & $\begin{array}{l}\text { Ischemia/ } \\
\text { Hypoxia }^{a}\end{array}$ & $\begin{array}{l}\text { Reperfusion/ } \\
\text { Reoxygenation }^{\text {a }}\end{array}$ & $\begin{array}{c}\text { Autophagy Modulation } \\
\text { Method }\end{array}$ & Autophagy & $\begin{array}{l}\text { Proposed } \\
\text { Role }\end{array}$ \\
\hline $\begin{array}{l}\text { Funk \& Schnellmann }{ }^{13} \\
\text { (2012) }\end{array}$ & Mouse & $20 \mathrm{~min}$ & $24,72,144 \mathrm{~h}$ & - & Increased & NS \\
\hline Li et $\mathrm{al}^{14}(2014)$ & Mouse & $45 \mathrm{~min}$ & $\begin{array}{l}8,16,24 \\
72 \mathrm{~h} ; 7 \mathrm{~d}\end{array}$ & - & Increased & NS \\
\hline Chien et $\mathrm{al}^{8}$ (2007) & Rat & $45 \min$ & $4 \mathrm{~h}$ & Adenoviral delivery of $\mathrm{Bcl}-\mathrm{X} \mathrm{L}$ & Increased & Detrimental \\
\hline \multirow[t]{3}{*}{ Suzuki et $\mathrm{al}^{15}(2008)$} & HK-2 cells & $6,24 \mathrm{~h}$ & $10,12 h^{b}$ & $\begin{array}{l}\text { 3-Methyladenine, E64d/ } \\
\text { pepstatin A, siRNA } \\
\text { against Atg7 }\end{array}$ & $\begin{array}{l}\text { Hypoxia: } \\
\text { increased; } \\
\mathrm{H}_{2} \mathrm{O}_{2}: \mathrm{NS}\end{array}$ & Detrimental \\
\hline & Mouse & $30 \mathrm{~min}$ & $6,24 \mathrm{~h}$ & - & Increased & NS \\
\hline & $\begin{array}{r}\text { Human Tx } \\
\text { biopsies }\end{array}$ & Various & $1 \mathrm{mo}$ & - & Increased & NS \\
\hline Isaka et $\mathrm{al}^{9}$ (2009) & Mouse & $45 \mathrm{~min}$ & $48,96 \mathrm{~h}$ & Bcl-2 overexpression & Increased & Detrimental \\
\hline Wu et $\mathrm{al}^{10}(2009)$ & Rat & $60 \mathrm{~min}$ & $4,24 \mathrm{~h}$ & Ischemic preconditioning & Increased & Detrimental \\
\hline Yeh et $\mathrm{al}^{11}$ (2010) & Rat & $45 \mathrm{~min}$ & $4,6,12,24 \mathrm{~h}$ & $\begin{array}{l}\text { Repetitive hypoxic } \\
\text { preconditioning }\end{array}$ & Increased & Detrimental \\
\hline Turkmen et al ${ }^{16}$ (2011) & Mouse & $48 \mathrm{~h}$ (cold) & NA & Bafilomycin A1 & Increased & Detrimental \\
\hline Nakagawa et al ${ }^{12}$ (2012) & Rat & $40 \mathrm{~min}$ & $1,2,4,7 d$ & Everolimus & Decreased & Detrimental \\
\hline \multirow{2}{*}{ Jiang et $\mathrm{al}^{2}(2010)$} & Rat RPTCs & $2 \mathrm{~h}$ & $2 \mathrm{~h}$ & siRNA against Beclin 1, Atg5 & Increased & Protective \\
\hline & Mouse & $30 \mathrm{~min}$ & $6,24,48 \mathrm{~h}$ & $\begin{array}{l}\text { Chloroquine, 3- } \\
\text { methyladenine }\end{array}$ & Increased & Protective \\
\hline Kimura et $\mathrm{al}^{3}$ (2011) & Mouse & $40 \mathrm{~min}$ & $\begin{array}{l}3,6,12,24 \\
\quad 48 \mathrm{~h}\end{array}$ & Atg5 knockout & Increased & Protective \\
\hline Liu et $\mathrm{al}^{4}(2012)$ & Mouse & $25 \mathrm{~min}$ & $24 \mathrm{~h}$ & Atg5 knockout & Increased & Protective \\
\hline Jiang et $\mathrm{al}^{5}(2012)$ & Mouse & $25 \min$ & $24 \mathrm{~h}$ & Atg7 knockout & NS & Protective \\
\hline Ishihara et $\mathrm{al}^{17}$ (2013) & $\begin{array}{l}\text { NRK-52E } \\
\text { cells }\end{array}$ & $2,4,6 \mathrm{~h}$ & $4,8,12 h^{c}$ & $\begin{array}{l}\text { BNIP3 and sestrin siRNA } \\
\text { and overexpression }\end{array}$ & Increased & Protective \\
\hline Lempiainen et $\mathrm{al}^{6}$ (2013) & Rat & $40 \mathrm{~min}$ & $24 \mathrm{~h}$ & Caloric restriction & Decreased & Protective \\
\hline
\end{tabular}

Abbreviations: Bcl-XL, B-cell lymphoma-extra large; $\mathrm{H}_{2} \mathrm{O}_{2}$, hydrogen peroxide; HK-2 cells, human kidney 2 cells; IRI, ischemiareperfusion injury; NA, not applicable; NS, not specified; RPTC, renal proximal tubular cell; siRNA, small interfering RNA; Tx, transplant.

${ }^{a}$ Ischemia and reperfusion pertain to the in vivo models; hypoxia and reoxygenation, to the in vitro systems.

${ }^{\text {b}}$ Treatment with $500 \mu \mathrm{M}$ of $\mathrm{H}_{2} \mathrm{O}_{2}$.

${ }^{\mathrm{c}}$ Treatment with $400 \mu \mathrm{M}$ of $\mathrm{H}_{2} \mathrm{O}_{2}$.

estimated glomerular filtration rate of $45 \mathrm{~mL} / \mathrm{min} / 1.73 \mathrm{~m}^{2}$ as calculated using the CKD-EPI equation). ${ }^{20}$

A preimplantation biopsy at the end of cold ischemia showed mild acute tubular necrosis without significant chronic changes. Interestingly, p62 staining in the kidney structures was less intense than in a donor kidney without delayed graft function (Fig 1). This low p62 expression suggests abnormally elevated autophagy (discussed next) and calls into question whether and how kidney autophagy affects early transplant function after kidney transplantation

\section{PATHOGENESIS}

\section{What Is Autophagy?}

\section{Overview}

At least 3 different mechanisms of autophagic degradation exist: microautophagy, chaperone-mediated autophagy, and macroautophagy. ${ }^{21}$ Macroautophagy, the best studied form and hereafter referred to simply as autophagy, involves the transport of cytoplasmic material in double-membranous vesicles (autophagosomes) to lysosomes for degradation and recycling (Fig 2A). Although first described in 1963 by Christian de Duve, autophagy research only flourished after the key autophagy gene BECN1 (which encodes beclin 1) was identified as a tumor suppressor in 1999. ${ }^{22,23}$ Further research established its vital role in cellular homeostasis and viability and revealed its significance in a plethora of continuously expanding disorders in different tissues. ${ }^{24}$ For example, mutations or disorders in autophagy have been linked with Crohn disease, systemic lupus erythematosus, cancers, and neurodegenerative diseases, among others. $^{25}$

\section{Autophagy as a Key Regulator of Cellular Life and Death}

In the absence of stress, constitutive autophagic activity ensures cellular homeostasis by mediating the turnover of macromolecules and organelles. In rapidly proliferating cells, this mechanism is generally dispensable because cell division dilutes cellular material into daughter cells, preventing the accumulation of old or damaged components. However, in differentiated or senescent cells such as neurons, (cardio) myocytes, and podocytes, basal levels of autophagy become more important. As such, perturbations in autophagy are involved in cellular degeneration (eg, neurodegenerative diseases and cardiomyopathies) ${ }^{24}$ characterized by accumulations of protein aggregates 

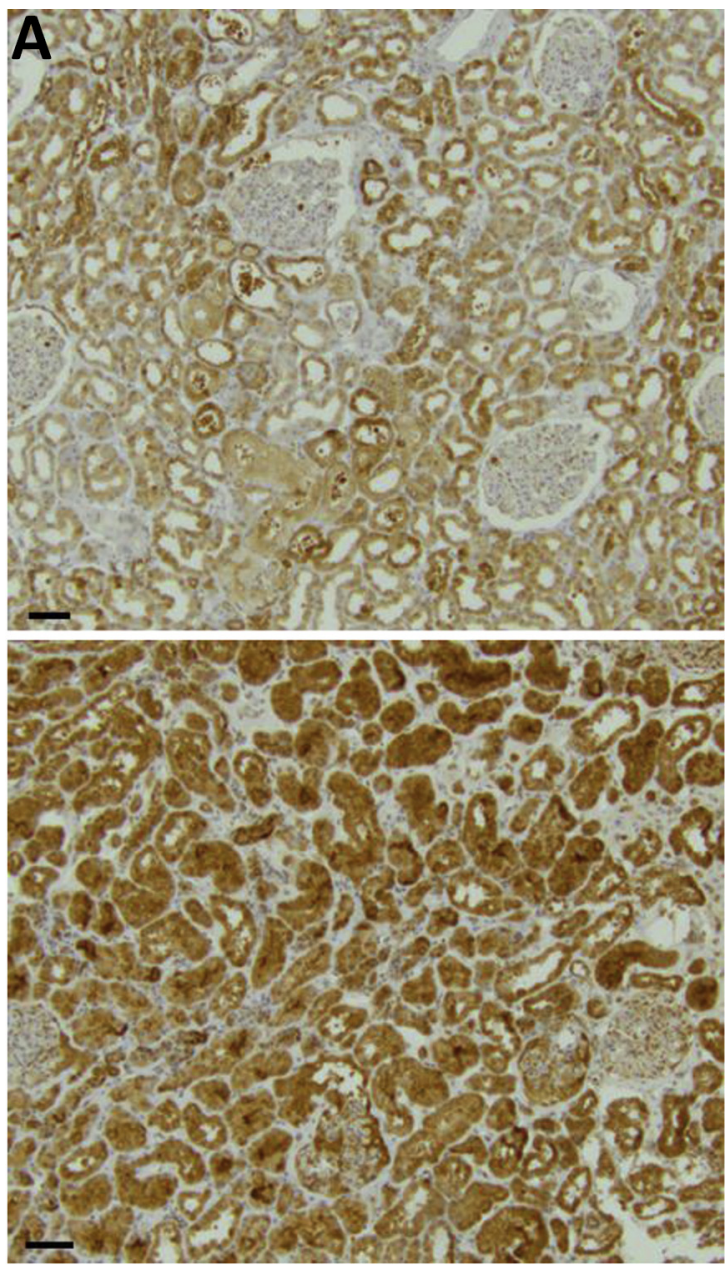

B

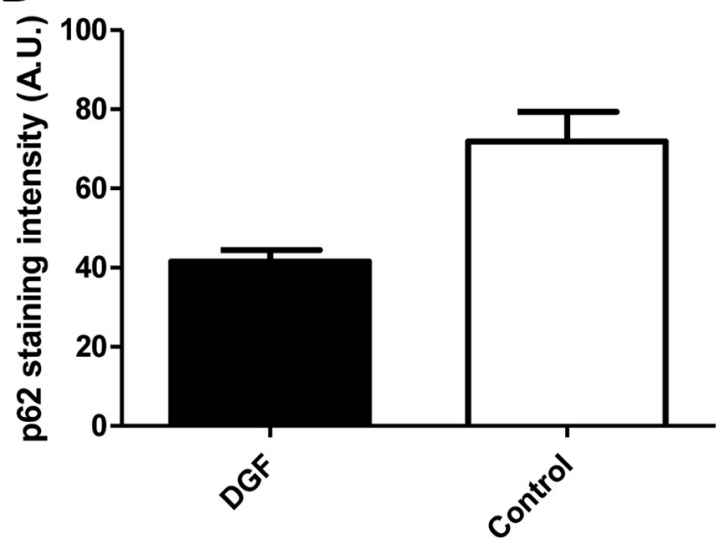

Figure 1. p62 staining on preimplantation biopsy specimens from donor kidneys. (A) (Top) p62 staining performed on a preimplantation biopsy specimen of a kidney that developed delayed graft function (DGF); (bottom) example of typical p62 staining, performed on a preimplantation biopsy specimen of a kidney that did not develop DGF. Donors and recipients were age- and sex-matched and staining was performed on both sections in the same conditions simultaneously. Pictures were taken with the same microscope settings and parameters. Scale bar represents $100 \mu \mathrm{m}$. (B) Quantification of the intensity of p62 staining of the DGF and control case. The histogram represents mean intensity (corrected for the background) \pm standard deviation of 15 to 20 fields of each analyzed section. Quantification was performed using ImageJ. and damaged mitochondria. Because of its vital role, whole-body knockout of autophagy leads to early (embryonic or neonatal) death in mice, and conditional (tissue-specific) knockout leads to severe pathologies in the affected tissue. ${ }^{26}$

When cells are under stress, autophagy is stimulated to facilitate the recycling of (damaged) macromolecules, organelles, or protein aggregates into cellular building blocks that are incorporated in antistress responses and energy production. As such, autophagy promotes cellular survival and counteracts stress-induced cell death, justifying its therapeutic potential in stress-related disorders. However, autophagy is also suggested to provoke cell death, although the mechanisms behind it have been a matter of debate. ${ }^{27-29}$ Possible mechanisms include the enhancement of apoptosis by autophagy protein fragments, ${ }^{30}$ autophagic degradation of antioxidant catalases resulting in increased reactive oxygen species production, ${ }^{31}$ or autophagy-dependent lysosomal membrane permeabilization, but these remain to be demonstrated. ${ }^{32}$ Recently, a cell-permeable peptide corresponding in sequence to a portion of the autophagy protein beclin 1 was found to trigger a novel necrotic-like cell death (termed autosis) in different cellular models, which was prevented by inhibition of autophagy. Autosis was also observed in a small subpopulation of nutrient-deprived cells $(\sim 1 \%)$ and in vivo in a mouse model of cerebral ischemia. ${ }^{33}$ Interestingly, beclin 1 is inhibited by the antiapoptotic $\mathrm{Bcl}-2$ proteins (eg, $\mathrm{Bcl}-2$ and $\mathrm{Bcl}-\mathrm{X}_{\mathrm{L}}$; Fig 2A), and cell death is induced when beclin 1 lacks the Bcl-2-binding motif, ${ }^{34}$ suggesting that $\mathrm{Bcl}-2$ proteins are able to inhibit autophagy-dependent cell death. This Bcl-2-beclin 1 crosstalk thus represents an important pivot in the cellular life versus death decision.

\section{Molecular Regulation of Autophagy}

Many autophagy proteins (Atg proteins) constitute the molecular machinery of autophagy. ${ }^{35}$ Proteins relevant for this review are shown in Fig 2A.

Autophagy initiates with the formation of a doublemembranous structure (phagophore) that elongates into a vesicle (autophagosome). Two protein complexes are essential in phagophore formation: the ULK1/2 complex and the class III phosphatidylinositol 3-kinase (PtdIns3KC3) complex.

Phosphorylation of ULK1/2 complex members by the mammalian target of rapamycin (mTOR) inhibits the complex and blocks autophagy. As such, mTOR suppression by nutrient deprivation or chemically (eg, rapamycin or everolimus) stimulates autophagic recycling. Interestingly, this causes a negative feedback: the newly formed amino acids reactivate mTOR and suppress autophagy, which is partially 


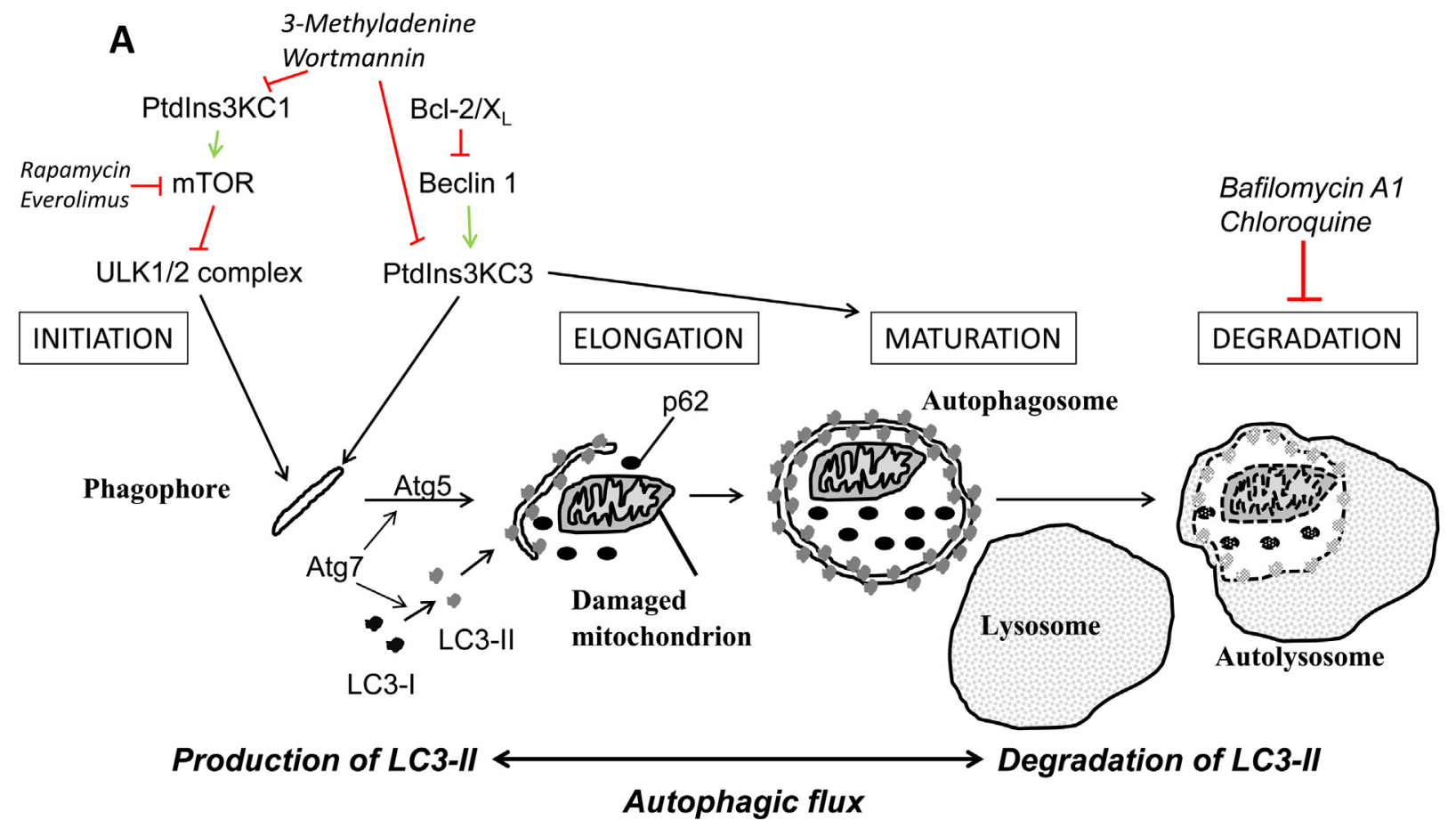

B
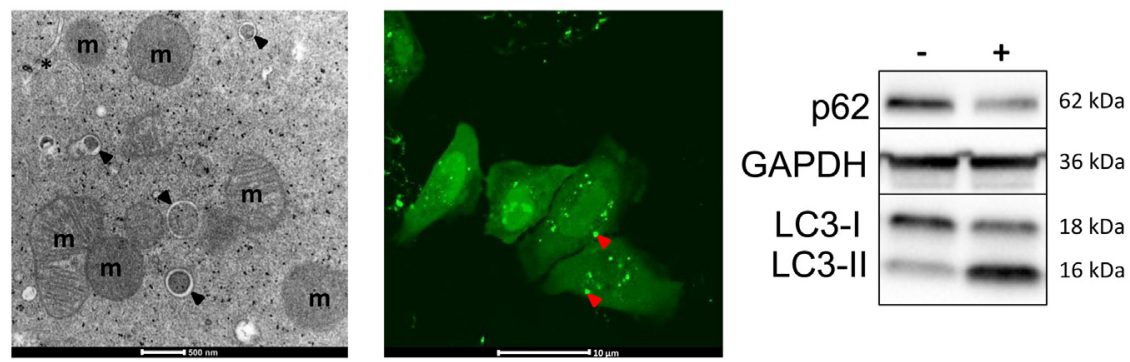

Figure 2. Overview of autophagy and the common monitoring techniques. (A) Overview of molecular regulation of the autophagy process and its modulators. See text for details. (B) The most frequently used techniques to measure autophagy. (Left) Transmission electron microscopy is still the gold standard to visualize autophagosomes (arrows). Scale bar, $500 \mathrm{~nm}$. (Middle) Fluorescently labeled LC3 (either through immunofluorescence or tagged with green fluorescent protein) appears as fluorescent punctae due to the accumulation of LC3-II at the autophagosomal membranes. Each puncta represent an autophagosome (arrows). Scale bar, $10 \mu \mathrm{m}$. (Right) LC3-I and LC3-II migrate at different rates in a protein gel, consistent with different apparent molecular weights. Stimulation of autophagy (indicated with +; - represents control conditions) results in an increase in LC3-II. Concomitantly, levels of the autophagy cargo protein $\mathrm{p} 62$ decrease. GAPDH represents the loading control. Abbreviation: $\mathrm{m}$, mitochondria.

responsible for the dynamic, transient, or fluctuating activity that autophagy often displays during prolonged stress. ${ }^{36}$ The PtdIns3KC3 complex requires beclin 1 in order to generate PtdIns-3-phosphate on endoplasmic reticulum membranes at specific sites where phagophore formation will initiate. ${ }^{37}$ Beclin 1 and PtdIns3KC3 are also involved in the maturation phase of the autophagic pathway (Fig 2A).

Phagophore elongation requires formation of the Atg12-Atg5-Atg16 complex and lipidation of LC3-I to phosphatidylethanolamine-conjugated LC3 or LC3-II. Both reactions are in part mediated by Atg7. LC3 conversion is used to study autophagy (Fig 2B): LC3-I and LC3-II migrate differently during gel electrophoresis and are detected as 2 separate bands in Western blots. The band intensity of the autophagic form LC3-II reflects autophagic activity. Furthermore, because LC3-II concentrates at autophagosomal membranes, autophagosomes can be visualized by fluorescently labeling LC3 .

Importantly, LC3-II is also degraded inside the lysosomes. As such, the amount of LC3-II at a particular time is dependent on both the autophagosome production and degradation rate. This continuous turnover (termed autophagic flux) complicates the value of LC3-II as an autophagy marker and advocates the need for additional markers, such as the autophagy degradation substrate Sqstm1/p62 (Figs 1 and 2B). ${ }^{19}$

Finally, autophagosomes fuse with endosomes (maturation) and eventually with lysosomes, forming 
autolysosomes, in which the autophagic cargo is degraded by lysosomal enzymes.

\section{What Is the Role of Autophagy in the Normal Kidney?}

Before examining the role of autophagy during renal IRI, it is important to understand its physiologic role in the different renal cell types.

Podocytes are highly differentiated cells that rely heavily on autophagy and, in mice, display many autophagosomes. ${ }^{14,38}$ Consequently, p62 degradation in these cells is high and glomeruli have lower p62 levels than their neighboring tubular structures (Fig 1). Transgenic mice in which Atg5 is knocked out specifically in podocytes display increased endoplasmic reticulum stress and an accumulation of oxidized and ubiquitinated proteins, resulting in podocyte loss, proteinuria, and glomerulosclerosis. ${ }^{39}$ Cells of the collecting ducts also show high autophagy levels. ${ }^{14}$ In contrast, tubular cells contain limited autophagosomes in mice. Starvation strongly increases their number, suggesting that autophagy is important for tubular cell survival. ${ }^{14,38}$ Proximal tubular cells consume a large amount of energy during electrolyte reabsorption, and autophagy is probably important for their energy needs and mitochondrial turnover. Moreover, proximal tubular cells have high lysosomal activity necessary for protein degradation. This is reflected by the development of glycosuria and reduced kidney function in proximal tubule-specific Atg5 knockout mice. $^{3,4}$ Distal tubular cells, whose function is more passive and less energy dependent, rely less on autophagy for their homeostasis. Distal tubulespecific Atg5 knockout mice have no decreased kidney function despite an increase in levels of oxidative markers. ${ }^{4}$ The likely more important role of autophagy in proximal versus distal tubular cells might explain the higher sensitivity of proximal tubular cells to IRI compared with distal tubular cells.

\section{Is the Role of Autophagy in Renal IRI Protective or Detrimental?}

The consecutive hypoxic and oxidative stress evoked by ischemia and reperfusion has been shown to enhance autophagy in different rodent models of renal IRI. ${ }^{2-4,8-11,13-15}$ However, suppressed autophagy has also been observed in 2 rat models subjected to 40 minutes of warm renal ischemia (Table 1). ${ }^{6,12}$ After 48 hours of cold ischemia, autophagy is increased. ${ }^{16}$ Regardless of its dynamics (increased or decreased), the role of autophagy after renal IRI is a major matter of debate, with both protective and detrimental properties proposed (Table 1).

\section{Evidence That Autophagy Ameliorates Renal IRI}

Considering its predominantly protective properties, it would make sense that autophagy stimulation attenuates renal IRI. In this regard, autophagy suppression by the PtdIns3K inhibitor 3methyladenine (3-MA) or by small interfering RNA (siRNA) against beclin 1 or Atg5 transcripts increases apoptosis in vitro in rat proximal tubular cells subjected to hypoxia-reoxygenation. In vivo, 3-MA and the lysosomal inhibitor chloroquine aggravate the loss of renal cells 48 hours postreperfusion in a 30-minute bilateral renal ischemia mouse model. ${ }^{2}$ Similar effects were observed after 25 minutes of bilateral renal ischemia in autophagy-deficient (proximal tubulespecific Atg7 knockout) mice. ${ }^{5}$ Two independently generated proximal tubule-specific Atg5 knockout mouse models subjected to 25 or 35 minutes of bilateral renal ischemia have also been reported to display increased kidney damage, apoptosis, and accumulation of p62- and ubiquitin-positive inclusions. $^{3,4}$ Finally, caloric restriction starting 2 weeks prior to IRI (40 minutes of bilateral ischemia) also has been shown to protect rats from kidney damage. Caloric restriction is known to stimulate autophagy through the activation of nicotinamide adenine dinucleotide (NAD)-dependent deacetylase sirtuin $1 .^{40}$ Surprisingly, the protection against renal IRI was attributed to autophagy in a sirtuin 1-independent manner because 3-MA, but not the sirtuin 1 inhibitor sirtinol, abrogates the protective effect of caloric restriction. ${ }^{6}$

\section{Evidence That Autophagy Exacerbates Renal IRI}

Despite ample evidence of the protective properties of autophagy, detrimental effects have also been attributed to autophagy during renal IRI (Table 1). In uninephrectomized rats, the remaining kidney is protected from IRI ( 45 minutes of ischemia) by adenoviral delivery of the Bcl-2 family proteins Bcl-2 and Bcl$\mathrm{X}_{\mathrm{L}}$. This effect was suggested to be mediated by both the antiapoptotic and antiautophagic properties of these proteins. ${ }^{8}$ The protective effect of $\mathrm{Bcl}-2$ was also independently observed in transgenic mice overexpressing Bcl-2; when these animals are subjected to 45 minutes of bilateral ischemia, a decrease in both apoptotic and autophagic features is observed. ${ }^{9}$ Moreover, inhibition of autophagy by 3-MA, the lysosomal inhibitors pepstatin A/E64d, or siRNA against Atg7 protects against oxidative stress induced by hydrogen peroxide in vitro in proximal tubular HK2 (human kidney 2) cells. ${ }^{15}$ In vivo, the mTOR inhibitor everolimus stimulates autophagy in rat kidney and aggravates renal IRI after 40 minutes of bilateral ischemia. ${ }^{12}$ Finally, the protective effects of ischemic and repetitive hypoxic preconditioning were reported 
to be accompanied by a decrease of autophagy in 2 models of unilateral ischemia (for 45 and 60 minutes) in uninephrectomized rat kidneys. ${ }^{10,11}$

\section{Why Is It Difficult to Determine the Exact Role of Autophagy in Renal IRI?}

\section{Limitations of In Vitro Models}

In vitro simulation of IRI by hypoxia-reoxygenation $^{2}$ or treatment with hydrogen peroxide (Table 1) ${ }^{15,17}$ has the advantage of dissecting the effect of a single stress response in one cell type independent of other factors. However, in vivo, IRI is the result of oxidative stress accompanied by nutrient deprivation, loss of blood flow, and inflammatory and immune signals (Fig 3). Moreover, the multiple kidney cell types will react differently to in vivo renal IRI and influence each other through direct and indirect communication. This heterogeneity is absent in a cell culture system. Thus, the magnitude, kinetics, and outcome in vivo are dependent on the culmination of cell-intrinsic (ie, the molecular stress-activated pathways) and cell-extrinsic (ie, immune signals) factors that are lacking in vitro.

\section{Dynamic Nature of Autophagy}

As mentioned, autophagy often displays a transient dynamic nature, including in hypoxic rat renal proximal tubular cells, ${ }^{2}$ hydrogen peroxide-treated NRK-52E cells, ${ }^{17}$ and in vivo after 40 minutes of unilateral renal ischemia, ${ }^{3}$ in which an initial increase is followed by a decrease in autophagy markers. This dynamic nature of autophagy complicates the comparison of studies analyzing different durations of ischemia or reperfusion (Table 1). Ideally, therefore, multiple time points covering the entire IRI process need to be analyzed.

\section{Crosstalk of Autophagy With Other Cell Death Pathways}

Overexpression of Bcl-2 family proteins has been shown to aggravate renal IRI. ${ }^{8,9}$ Because Bcl-2 proteins are both anti-autophagic and anti-apoptotic, it is unclear whether the end result is due to autophagy or apoptosis suppression. Although apoptosis is usually considered to be harmful during IRI, it may nevertheless have beneficial effects as an alternative noninflammatory cell death pathway, in contrast to necrosis, and in the safe removal of damaged kidney tissue. In addition, autophagy and apoptosis are generally mutually exclusive. By removing damaged mitochondria, autophagy eliminates potential apoptotic mitochondrial outer membrane permeabilization events. Conversely, the apoptotic effectors calpains and caspases can cleave autophagy proteins and by this mechanism even turn them into proapoptotic proteins. ${ }^{30}$ The existence of this mutual exclusion in renal IRI is supported by the observation that proximal tubulespecific Atg5 knockout mice display more apoptosis after renal IRI or cisplatin treatment. ${ }^{3,41,42}$ This tight molecular crosstalk between autophagy and the other cell death pathways highlights the need to investigate all these processes in parallel (Fig 3).

\section{Absence of Specific Markers for Autophagy}

The most common autophagy markers (the number of autophagosomes or amount of LC3-II) do not represent autophagic flux very well. For example, lysosomal dysfunction will lead to accumulation of autophagosomes and LC3-II, which can be erroneously interpreted as autophagy stimulation. ${ }^{19}$ Consequently,
ISCHEMIA (warm/cold)

\section{REPERFUSION}

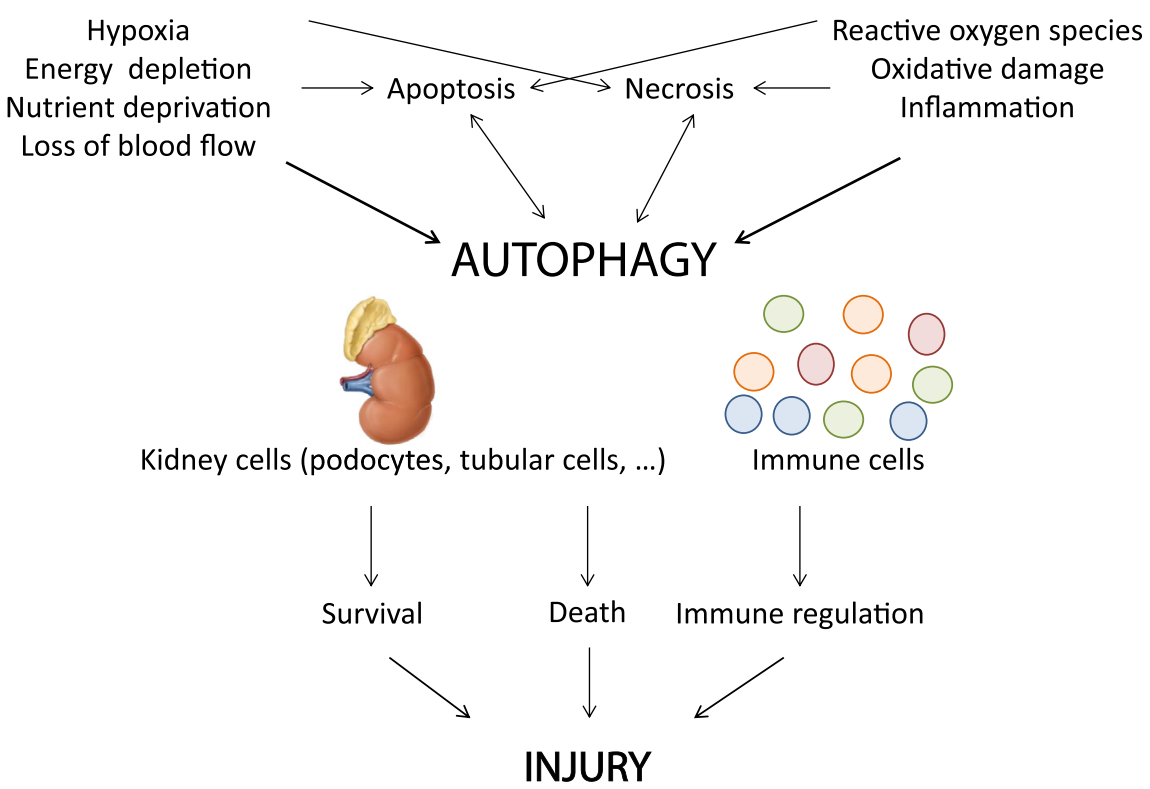

Figure 3. Difficulties in research on autophagy in renal ischemia-reperfusion injury. Ischemic stress consists of hypoxia, nutrient and energy depletion, and loss of blood flow, whereas during reperfusion, the oxidative stress and subsequent intracellular damage are supplemented by an inflammation factor. The autophagy response to all these stress factors occurs by different and overlapping pathways. In addition, ischemia and reperfusion affect apoptosis and necrosis, with which autophagy is known to have molecular crosstalk. Autophagy not only regulates the homeostasis and viability of the different local kidney cells, but also plays a role in circulating immune cells, where it fine-tunes (innate and adaptive) immune responses. The culmination of these responses eventually determines its effect on kidney injury. 
additional autophagy markers (such as Sqstm1/p62; Figs 1 and 2B) and measuring techniques are required to more correctly apprehend the autophagic flux.

Recently, a transgenic mouse model expressing LC3 double-tagged with red and green fluorescent protein (RFP-GFP-LC3) was developed. ${ }^{14}$ Unlike RFP, GFP is acid sensitive, meaning its fluorescence is rapidly quenched inside lysosomes. This behavior provides a way to differentiate autophagosomes (which fluoresce red and green) from autolysosomes (which fluoresce red only). In this model, 45 minutes of unilateral ischemia augments the number of red and green dots (increased autophagic flux) in proximal tubular cells, followed by enhanced autophagosome clearance (mostly red dots) 3 days postreperfusion. ${ }^{14}$ This transgenic mouse model will have great value in increasing understanding of the autophagic flux during renal IRI in different experimental conditions.

\section{Limitations of Autophagy Modulators}

The chemical autophagy modulators that are used to study the role of autophagy in renal IRI have multiple side effects on other related intracellular pathways. mTOR inhibitors (eg, rapamycin and everolimus) stimulate autophagy, but also inhibit cell growth, proliferation, and protein transcription and translation. PtdIns3K inhibitors (eg, 3-MA) inhibit autophagy through suppression of PtdIns3KC3, but also affect cell growth, proliferation, and the stability of hypoxiainducible factor $1 \alpha$ (HIF-1 $\alpha)$ through suppression of PtdIns3KC1. Finally, lysosomal inhibitors (eg, chloroquine and bafilomycin A1) hamper all lysosomedependent pathways. Consequently, it is premature to pinpoint the protective or detrimental effect of these compounds solely to autophagy modulation. Moreover, it is important to note that autophagy helps balance the inflammatory and both innate and adaptive immune responses through various mechanisms. ${ }^{43}$ It is thus unclear whether the effect of an autophagy modulator is caused by alterations in renal parenchymal cell viability or altered immune regulation (Fig 3). The complex regulation of adaptive immunity and immunosuppression by autophagy is thoroughly reviewed elsewhere. ${ }^{44}$

Interestingly, proximal tubule-specific Atg5 knockout mice or proximal tubule-specific Atg7 knockout mice permit kidney-specific inhibition of autophagy. ${ }^{3-5}$ In these models, IRI is aggravated, pointing toward a protective role for autophagy. However, it should be noted that the lack of proper autophagy completion induces an accumulation of unusual multiple tightly packed concentric membranes or cytoplasmic inclusion bodies after renal IRI ${ }^{4}$ which could have toxic properties and account for the observed exacerbation of the injury.

\section{RECENT ADVANCES}

\section{A Dual Role for Autophagy in Renal IRI}

In general, autophagy has shown protective properties in in vivo models when ischemic duration is limited (25-40 minutes), whereas detrimental effects have been observed when the period of ischemia is prolonged to 40 to 60 minutes (Table 1). We therefore hypothesize that autophagy can switch roles, depending on the severity of the ischemic injury and, for a given severity, the phase of the IRI process (early or late after initiation of IRI). Longer ischemic periods could increase the likelihood of tipping the balance toward autophagy-dependent cell death (Fig 4). The 40-minute ischemia mark may represent this tipping point, but will likely also depend on other factors such as unilateral versus bilateral ischemia and the type, strain, and sex of animal used. In support of this, autophagy and apoptosis were observed to be differently regulated in male versus female rats subjected to cardiac IRI. ${ }^{45}$ During cardiac IRI, a dual role for autophagy has already been proposed, albeit independent of ischemic duration. ${ }^{46}$ We thus hypothesize that this transition during reperfusion in the kidney partially accounts for the contradictory findings in the literature. The mechanisms by which this switch is set in motion are largely speculative, but probably depend on the survival versus death properties of beclin $1^{46}$ and its interaction with the Bcl-2 family proteins.

\section{Implications for Autophagy Modulation as a Therapeutic Strategy in Renal IRI}

The autophagy-independent effects of the standard autophagy modulators are inappropriate for therapeutic autophagy modulation in renal IRI because they will either act on kidney cell proliferation (eg, 3-MA and rapamcyin) or affect lysosomal function, both of which are likely too important to be disturbed during renal IRI. However, several mTOR and phosphoinositol-3-kinase (PI3K)-independent compounds (eg, trehalose and resveratrol) could potentially activate autophagy without affecting other vital functions. Resveratrol, for example, is an antioxidant that acts on sirtuin 1 and has protective effects after renal IRI. ${ }^{47}$ Interestingly, by acting independently of mTOR, trehalose can alleviate disruptions in the mTOR-dependent autophagic flux, as has been demonstrated in neurodegenerative diseases. ${ }^{48}$

Based on the proposed dual function of autophagy in renal IRI, optimal autophagy modulation should restrict autophagy levels inside a protective therapeutic window and depends on the extent of IRI (Fig 4). Upon severe ischemia, autophagy inhibitors should preferably outweigh the activators. In addition, screening for autophagy markers in a baseline donor biopsy specimen might be a valuable method to detect whether 


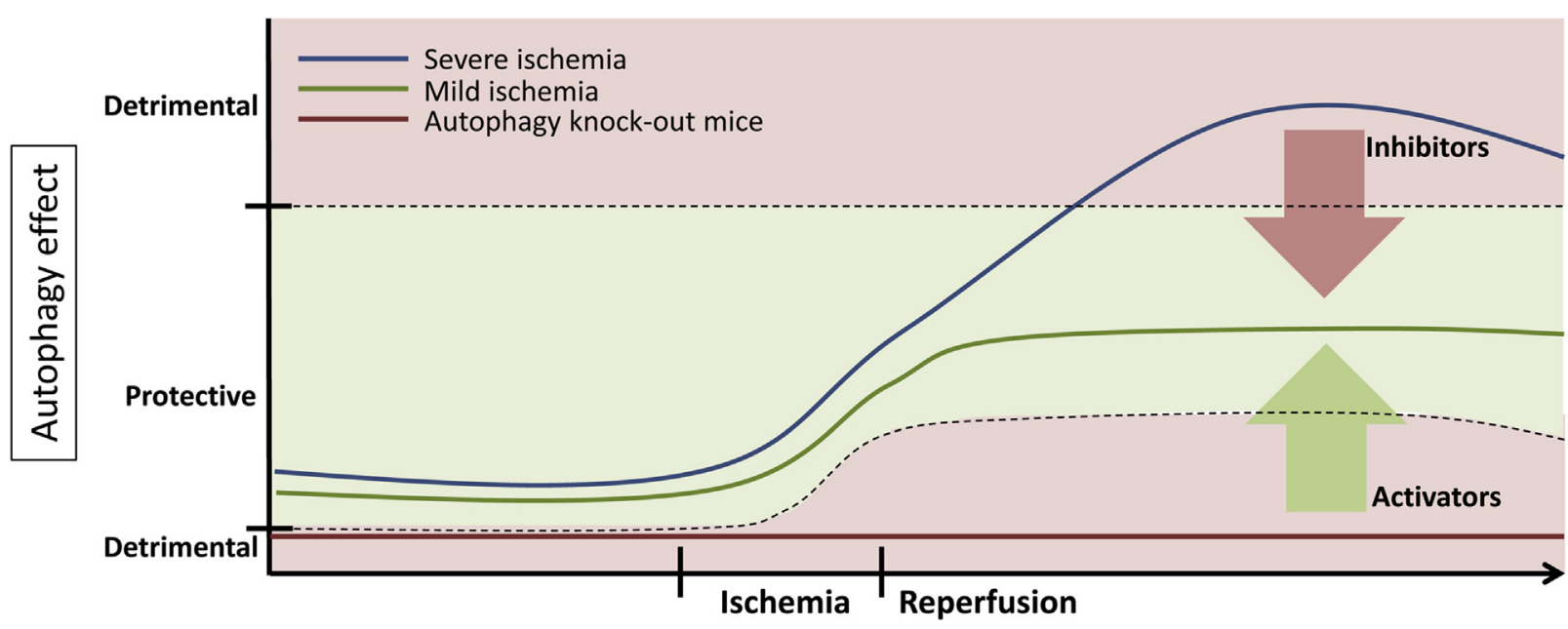

Figure 4. Hypothesis of the dual role of autophagy in renal ischemia-reperfusion injury (IRI) and the subsequent implications for therapy. Autophagy is a protective survival process in renal IRI, as long as its function is maintained in its protective window (green area). Upon ischemia-reperfusion, the lowest protective level of autophagy augments because autophagy upregulation is required for survival, narrowing the protective window (lower dashed line). Inhibition of autophagy below these levels will result in more damage in an extreme stress situation as IRI and thus is best avoided. This situation is created, for example, in the autophagy knockout mouse models (red line). Following mild ischemia, autophagy is likely upregulated within the limits of its protective window (green line). Severe ischemia increases the chance for autophagy to surpass its threshold (upper dashed line) and become detrimental during reperfusion (blue line). To modulate autophagy as a therapeutic strategy, it is advisable to stimulate and/or inhibit autophagy so that its levels are conducted inside the protective therapeutic window (red and green arrows).

autophagy is already deregulated pretransplantation, lowering the threshold for autophagy-dependent cell death posttransplantation. This information can be taken into account during postoperative (immunosuppressive) drug treatment. In our case vignette, for example, the decreased p62 expression could indicate overactivated autophagy preimplantation (Fig 1), arguing for the use of autophagy inhibitors instead of autophagy stimulators to improve transplant outcome.

\section{Modulation of Autophagy in Renal IRI With Diuretics and Immunosuppressants}

Several diuretics (furosemide) and immunosuppressive drugs (rapamycin, tacrolimus, MMF, and corticosteroids) used in the setting of AKI and kidney transplantation, respectively, are autophagy modulators (Table 2), ${ }^{49-65}$ which could partially explain their influences on renal IRI. Furosemide is often applied in an attempt to promote diuresis in AKI. Interestingly, in rat kidneys, furosemide induces the number of autophagosomes in the thick ascending loop of Henle, but not in proximal tubular cells. ${ }^{49}$ The autophagystimulating properties could partially explain the conflicting results regarding the use of furosemide in rat IRI models subjected to unilateral versus bilateral clamping; after unilateral IRI, furosemide improves outcomes and decreases apoptosis, ${ }^{66,67}$ whereas it increases kidney function and lethality in a bilateral renal IRI model. ${ }^{68}$ Based on our hypothesis on the dual role of autophagy depending on the extent of the ischemic stress, furosemide-induced autophagy will therefore more likely induce detrimental autophagy after bilateral, with higher IRI, than unilateral clamping. Moreover, the detrimental effect of furosemide only occurs in adult, not young, rats. ${ }^{68}$ Aging is often characterized by disturbances in the autophagic pathway, which may already lower the threshold for detrimental autophagy. This agedependent autophagic predysfunction, together with the high ischemic stress during AKI, may explain the harmful effects of furosemide observed in humans and the subsequent current debate on the use of diuretics in the prevention or treatment of AKI. ${ }^{69}$

Immunosuppressants will also affect autophagy after kidney transplantation, with consequences for IRI and rejection. The latter is beyond the scope of this review and is reviewed elsewhere. ${ }^{44}$ Rapamycin is reported to cause both better and worse outcomes in rodent kidney IRI and transplantation models. ${ }^{70-75}$ These effects are generally attributed to its antiproliferative properties, but it is unclear whether autophagy stimulation is also involved. The exact outcome also likely depends on the administered dose, ${ }^{76}$ ischemic time, markers measured, and phase postreperfusion (Fig 4). ${ }^{77,78}$ For example, the rapamycin analogue everolimus increases interleukin 6 , but reduces TNF- $\alpha$ (tumor necrosis factor $\alpha$ ) levels in a rat IRI model and increases myeloperoxidase staining 2 hours postreperfusion, but reduces it 24 hours postreperfusion. ${ }^{77}$ These findings highlight again the analysis of multiple time points during the entire IRI process (see previous discussion).

Interestingly, in a rat kidney transplantation model, protective effects of rapamycin are enhanced when 
Table 2. Overview of the Most Commonly Used Drugs in AKI and Kidney Transplantation and Their Effect on Autophagy

\begin{tabular}{|c|c|c|}
\hline Compound & Effect on Autophagy & Reference \\
\hline \multicolumn{3}{|c|}{ Diuretics } \\
\hline Furosemide & $\begin{array}{l}\text { Stimulation in loop of Henle, } \\
\text { not in proximal tubular cells } \\
\text { Immunosuppressants }\end{array}$ & 49 \\
\hline Rapamycin & Stimulation in vitro and in vivo & 50 \\
\hline Tacrolimus & $\begin{array}{l}\text { Stimulation in vivo in mouse } \\
\text { brain }\end{array}$ & 51 \\
\hline $\begin{array}{l}\text { Mycophenolate } \\
\text { mofetil }\end{array}$ & $\begin{array}{l}\text { Stimulation of chaperone- } \\
\text { mediated autophagy in vitro } \\
\text { in hepatocytes }\end{array}$ & 52 \\
\hline \multirow[t]{5}{*}{ Dexamethasone } & $\begin{array}{l}\text { Stimulation in vitro in } \\
\text { thymoma lymphocytes }\end{array}$ & 53 \\
\hline & $\begin{array}{l}\text { Stimulation of autophagy- } \\
\text { dependent cell death } \\
\text { in vitro in lymphoid } \\
\text { leukemia cells }\end{array}$ & 54 \\
\hline & $\begin{array}{l}\text { Stimulation in vivo in rat } \\
\text { soleus muscle }\end{array}$ & 55 \\
\hline & $\begin{array}{l}\text { Stimulation in vitro in primary } \\
\text { osteocytes }\end{array}$ & 56 \\
\hline & $\begin{array}{l}\text { Stimulation in vitro in } \\
\text { chondrocytes }\end{array}$ & 57 \\
\hline \multirow[t]{2}{*}{ Methylprednisolone } & $\begin{array}{l}\text { Stimulation in vivo in mouse } \\
\text { osteocytes }\end{array}$ & 58 \\
\hline & $\begin{array}{l}\text { Inhibition of autophagy- } \\
\text { dependent cell death in vivo } \\
\text { in rat spinal cord }\end{array}$ & 59 \\
\hline Azathioprine & $\begin{array}{l}\text { Stimulation in vitro in } \\
\text { hepatoblastoma cells }\end{array}$ & 60 \\
\hline \multirow[t]{5}{*}{ Cyclosporine A } & $\begin{array}{l}\text { Stimulation in vivo in rat } \\
\text { acinar cells }\end{array}$ & 61 \\
\hline & $\begin{array}{l}\text { Stimulation in vitro in rat } \\
\text { pituitary cells }\end{array}$ & 62 \\
\hline & $\begin{array}{l}\text { Inhibition of autophagic flux } \\
\text { in vivo in mouse kidney }\end{array}$ & 63 \\
\hline & $\begin{array}{l}\text { Stimulation of autophagy- } \\
\text { dependent cell death } \\
\text { in vitro in glioma cells }\end{array}$ & 64 \\
\hline & $\begin{array}{l}\text { Stimulation of autophagy- } \\
\text { dependent cell death } \\
\text { in vitro in rat pituitary cells }\end{array}$ & 65 \\
\hline
\end{tabular}

Abbreviation: AKI, acute kidney injury.

combined with tacrolimus or MMF. ${ }^{79,80}$ These immunosuppressants mostly have protective properties on IRI through anti-apoptotic or anti-inflammatory mechanisms. $^{72,81-83}$ In addition, tacrolimus also stimulates autophagy in vivo in the brain of mice, ${ }^{51}$ while MMF is a potent inducer of chaperonemediated autophagy, a special form of autophagy specific for degradation of oxidized/misfolded proteins mediated by heat shock proteins. ${ }^{52}$ These chaperones are upregulated during cardiac IRI, ${ }^{84}$ but a role for chaperone-mediated autophagy in renal IRI has not been studied yet. Nevertheless, it is possible that the beneficial effects of tacrolimus and MMF on renal IRI are partially caused by their ability to stimulate autophagy.
The widely used immunosuppressant cyclosporine A has also been identified as a strong modulator of autophagy in vitro and in vivo (Table 2). Interestingly, its long-term nephrotoxic effects have even been attributed to inhibition of the autophagic flux, leading to an accumulation of autophagosomes, ${ }^{63}$ and this mechanism could account for cyclosporine Ainduced autophagy-dependent cell death observed in glioma and pituitary cell models in vitro. ${ }^{64,65}$

One final note is that corticosteroids also affect autophagy. Although some glucocorticoids (eg, dexamethasone) stimulate autophagy in vitro and in vivo, methylprednisolone, the most commonly used steroid in transplantation, suppresses autophagy in the rat spinal cord and thereby alleviates spinal cord injury, suggesting that methylprednisolone can counteract autophagydependent cell death. ${ }^{59}$ In contrast, low doses of prednisolone stimulate autophagy, while high doses induce apoptosis in osteocytes in vivo. ${ }^{58}$ Whether these steroids enact similar effects on autophagy in kidney cells during renal IRI is an important future question to be addressed.

\section{SUMMARY}

Autophagy is an essential process situated in the center of the cellular life and death balance. As such, it has connections with many cellular pathways and is affected by various external compounds. Its duality highlights the need for prudence in autophagy data interpretation: fluctuating dynamics, lack of specific markers and modulators, its role in immunity, and the crosstalk with other cell death pathways all impede proper autophagy analysis. Future careful research is needed to improve understanding of the autophagic transition from protective to detrimental, opening new avenues to specifically modulate the detrimental properties of autophagy without affecting its survival function. Considering the autophagy-modulating properties of immunosuppressive drugs, their perioperative use needs to be revisited in light of the dual role of autophagy in renal IRI.

\section{ACKNOWLEDGEMENTS}

Support: None.

Financial Disclosure: The authors declare that they have no relevant financial interests.

\section{REFERENCES}

1. Bellomo R, Kellum JA, Ronco C. Acute kidney injury. Lancet. 2012;380(9843):756-766.

2. Jiang M, Liu K, Luo J, Dong Z. Autophagy is a renoprotective mechanism during in vitro hypoxia and in vivo ischemiareperfusion injury. Am J Pathol. 2010;176(3):1181-1192.

3. Kimura T, Takabatake Y, Takahashi A, et al. Autophagy protects the proximal tubule from degeneration and acute ischemic injury. J Am Soc Nephrol. 2011;22(5):902-913.

4. Liu S, Hartleben B, Kretz O, et al. Autophagy plays a critical role in kidney tubule maintenance, aging and ischemia-reperfusion injury. Autophagy. 2012;8(5):826-837. 
5. Jiang M, Wei Q, Dong G, Komatsu M, Su Y, Dong Z. Autophagy in proximal tubules protects against acute kidney injury. Kidney Int. 2012;82(12):1271-1283.

6. Lempiainen J, Finckenberg P, Mervaala EE, Sankari S, Levijoki J, Mervaala EM. Caloric restriction ameliorates kidney ischaemia/reperfusion injury through PGC-1alpha-eNOS pathway and enhanced autophagy. Acta Physiol (Oxf). 2013;208(4):410-421.

7. Pallet N, Livingston M, Dong Z. Emerging functions of autophagy in kidney transplantation. Am J Transplant. 2013;14(1):13-20.

8. Chien CT, Shyue SK, Lai MK. Bcl-xL augmentation potentially reduces ischemia/reperfusion induced proximal and distal tubular apoptosis and autophagy. Transplantation. 2007;84(9):1183-1190.

9. Isaka Y, Suzuki C, Abe T, et al. Bcl-2 protects tubular epithelial cells from ischemia/reperfusion injury by dual mechanisms. Transplant Proc. 2009;41(1):52-54.

10. Wu HH, Hsiao TY, Chien CT, Lai MK. Ischemic conditioning by short periods of reperfusion attenuates renal ischemia/ reperfusion induced apoptosis and autophagy in the rat. J Biomed Sci. 2009;16:19.

11. Yeh CH, Hsu SP, Yang CC, Chien CT, Wang NP. Hypoxic preconditioning reinforces HIF-alpha-dependent HSP70 signaling to reduce ischemic renal failure-induced renal tubular apoptosis and autophagy. Life Sci. 2010;86(3-4):115-123.

12. Nakagawa S, Nishihara K, Inui K, Masuda S. Involvement of autophagy in the pharmacological effects of the mTOR inhibitor everolimus in acute kidney injury. Eur $J$ Pharmacol. 2012;696(1-3):143-154.

13. Funk JA, Schnellmann RG. Persistent disruption of mitochondrial homeostasis after acute kidney injury. Am J Physiol Renal Physiol. 2012;302(7):F853-F864.

14. Li L, Wang ZV, Hill JA, Lin F. New autophagy reporter mice reveal dynamics of proximal tubular autophagy. J Am Soc Nephrol. 2014;25(2):305-315.

15. Suzuki C, Isaka Y, Takabatake Y, et al. Participation of autophagy in renal ischemia/reperfusion injury. Biochem Biophys Res Commun. 2008;368(1):100-106.

16. Turkmen K, Martin J, Akcay A, et al. Apoptosis and autophagy in cold preservation ischemia. Transplantation. 2011;91(11):1192-1197.

17. Ishihara M, Urushido M, Hamada K, et al. Sestrin-2 and BNIP3 regulate autophagy and mitophagy in renal tubular cells in acute kidney injury. Am J Physiol Renal Physiol. 2013;305(4):F495-F509.

18. Decuypere JP, Pirenne J, Jochmans I. Autophagy in renal ischemia-reperfusion injury: friend or foe? Am J Transplant. 2014;14(6):1464-1465.

19. Klionsky DJ, Abdalla FC, Abeliovich H, et al. Guidelines for the use and interpretation of assays for monitoring autophagy. Autophagy. 2012;8(4):445-544.

20. Levey AS, Stevens LA, Schmid CH, et al. A new equation to estimate glomerular filtration rate. Ann Intern Med. 2009;150(9):604-612.

21. Todde V, Veenhuis M, van der Klei IJ. Autophagy: principles and significance in health and disease. Biochim Biophys Acta. 2009;1792(1):3-13.

22. Liang $\mathbf{X H}$, Jackson $\mathrm{S}$, Seaman $\mathbf{M}$, et al. Induction of autophagy and inhibition of tumorigenesis by beclin 1. Nature. 1999;402(6762):672-676.

23. Klionsky DJ. Autophagy: from phenomenology to molecular understanding in less than a decade. Nat Rev Mol Cell Biol. 2007;8(11):931-937.

24. Choi AM, Ryter SW, Levine B. Autophagy in human health and disease. N Engl J Med. 2013;368(7):651-662.

25. Jiang P, Mizushima N. Autophagy and human diseases. Cell Res. 2014;24(1):69-79.
26. Marino G, Madeo F, Kroemer G. Autophagy for tissue homeostasis and neuroprotection. Curr Opin Cell Biol. 2011;23(2): 198-206.

27. Kroemer G, Levine B. Autophagic cell death: the story of a misnomer. Nat Rev Mol Cell Biol. 2008;9(12):1004-1010.

28. Shen S, Kepp O, Kroemer G. The end of autophagic cell death? Autophagy. 2012;8(1):1-3.

29. Clarke PG, Puyal J. Autophagic cell death exists. Autophagy. 2012;8(6):867-869.

30. Wirawan E, Vande Walle L, Kersse K, et al. Caspase-mediated cleavage of Beclin-1 inactivates Beclin-1-induced autophagy and enhances apoptosis by promoting the release of proapoptotic factors from mitochondria. Cell Death Dis. 2010;1:e18.

31. Yu L, Wan F, Dutta S, et al. Autophagic programmed cell death by selective catalase degradation. Proc Natl Acad Sci U S A. 2006;103(13):4952-4957.

32. Green DR, Galluzzi L, Kroemer G. Cell biology. Metabolic control of cell death. Science. 2014;345(6203):1250256.

33. Liu Y, Shoji-Kawata S, Sumpter RM Jr, et al. Autosis is a $\mathrm{Na}^{+}, \mathrm{K}^{+}$-ATPase-regulated form of cell death triggered by autophagy-inducing peptides, starvation, and hypoxia-ischemia. Proc Natl Acad Sci U S A. 2013;110(51):20364-20371.

34. Pattingre S, Tassa A, Qu X, et al. Bcl-2 antiapoptotic proteins inhibit Beclin 1-dependent autophagy. Cell. 2005;122(6): 927-939.

35. Ravikumar B, Sarkar S, Davies JE, et al. Regulation of mammalian autophagy in physiology and pathophysiology. Physiol Rev. 2010;90(4):1383-1435.

36. Decuypere JP, Welkenhuyzen K, Luyten T, et al. Ins $(1,4,5)$ $\mathrm{P} 3$ receptor-mediated $\mathrm{Ca}^{2+}$ signaling and autophagy induction are interrelated. Autophagy. 2011;7(12):1472-1489.

37. Axe EL, Walker SA, Manifava M, et al. Autophagosome formation from membrane compartments enriched in phosphatidylinositol 3-phosphate and dynamically connected to the endoplasmic reticulum. J Cell Biol. 2008;182(4):685-701.

38. Mizushima N, Yamamoto A, Matsui M, Yoshimori T, Ohsumi Y. In vivo analysis of autophagy in response to nutrient starvation using transgenic mice expressing a fluorescent autophagosome marker. Mol Biol Cell. 2004;15(3):1101-1111.

39. Hartleben B, Godel M, Meyer-Schwesinger C, et al. Autophagy influences glomerular disease susceptibility and maintains podocyte homeostasis in aging mice. J Clin Invest. 2010;120(4):1084-1096.

40. Morselli E, Maiuri MC, Markaki M, et al. Caloric restriction and resveratrol promote longevity through the sirtuin-1dependent induction of autophagy. Cell Death Dis. 2010;1:e10.

41. Takabatake Y, Kimura T, Takahashi A, Isaka Y. Autophagy and the kidney: health and disease. Nephrol Dial Transplant. 2014;29(9):1639-1647.

42. Takahashi A, Kimura T, Takabatake Y, et al. Autophagy guards against cisplatin-induced acute kidney injury. Am J Pathol. 2012;180(2):517-525.

43. Levine B, Mizushima N, Virgin HW. Autophagy in immunity and inflammation. Nature. 2011;469(7330):323-335.

44. Bizargity P, Schroppel B. Autophagy: basic principles and relevance to transplant immunity. Am J Transplant. 2014;14(8): 1731-1739.

45. Chen C, Hu LX, Dong T, et al. Apoptosis and autophagy contribute to gender difference in cardiac ischemia-reperfusion induced injury in rats. Life Sci. 2013;93(7):265-270.

46. Sciarretta S, Hariharan N, Monden Y, Zablocki D, Sadoshima J. Is autophagy in response to ischemia and reperfusion protective or detrimental for the heart? Pediatr Cardiol. 2011;32(3):275-281. 
47. Sener G, Tugtepe H, Yuksel M, Cetinel S, Gedik N, Yegen BC. Resveratrol improves ischemia/reperfusion-induced oxidative renal injury in rats. Arch Med Res. 2006;37(7):822-829.

48. Zhang X, Chen S, Song L, et al. mTOR-independent, autophagic enhancer trehalose prolongs motor neuron survival and ameliorates the autophagic flux defect in a mouse model of amyotrophic lateral sclerosis. Autophagy. 2014;10(4):588-602.

49. Bahro M, Gertig G, Pfeifer U. Short-term stimulation of cellular autophagy by furosemide in the thick ascending limb of Henle's loop in the rat kidney. Cell Tissue Res. 1988;253(3):625-629.

50. Inoki K. mTOR signaling in autophagy regulation in the kidney. Semin Nephrol. 2014;34(1):2-8.

51. Nakagaki T, Satoh K, Ishibashi D, et al. FK506 reduces abnormal prion protein through the activation of autolysosomal degradation and prolongs survival in prion-infected mice. Autophagy. 2013;9(9):1386-1394.

52. Bandyopadhyay U, Sridhar S, Kaushik S, Kiffin R, Cuervo AM. Identification of regulators of chaperone-mediated autophagy. Mol Cell. 2010;39(4):535-547.

53. Swerdlow S, McColl K, Rong Y, Lam M, Gupta A, Distelhorst CW. Apoptosis inhibition by Bcl-2 gives way to autophagy in glucocorticoid-treated lymphocytes. Autophagy. 2008;4(5):612-620.

54. Laane E, Tamm KP, Buentke E, et al. Cell death induced by dexamethasone in lymphoid leukemia is mediated through initiation of autophagy. Cell Death Differ. 2009;16(7):1018-1029.

55. Yamamoto D, Maki T, Herningtyas EH, et al. Branchedchain amino acids protect against dexamethasone-induced soleus muscle atrophy in rats. Muscle Nerve. 2010;41(6):819-827.

56. Xia X, Kar R, Gluhak-Heinrich J, et al. Glucocorticoid-induced autophagy in osteocytes. J Bone Miner Res. 2010;25(11):2479-2488.

57. Liu N, Wang W, Zhao Z, Zhang T, Song Y. Autophagy in human articular chondrocytes is cytoprotective following glucocorticoid stimulation. Mol Med Rep. 2014;9(6):2166-2172.

58. Jia J, Yao W, Guan M, et al. Glucocorticoid dose determines osteocyte cell fate. FASEB J. 2011;25(10):3366-3376.

59. Chen HC, Fong TH, Lee AW, Chiu WT. Autophagy is activated in injured neurons and inhibited by methylprednisolone after experimental spinal cord injury. Spine (Phila Pa 1976). 2012;37(6):470-475.

60. Hernandez-Breijo B, Monserrat J, Roman ID, et al. Azathioprine desensitizes liver cancer cells to insulin-like growth factor 1 and causes apoptosis when it is combined with bafilomycin A1. Toxicol Appl Pharmacol. 2013;272(3):568-578.

61. Bani D, Filipponi F, Magnani L, Houssin D, Romagnoli P. Morphological changes in rat pancreatic acinar cells induced by long-term treatment with cyclosporine and their reversal after withdrawal. Transplantation. 1990;50(5):830-834.

62. Yoo YM, Jeung EB. Melatonin suppresses cyclosporine Ainduced autophagy in rat pituitary GH3 cells. J Pineal Res. 2010;48(3):204-211.

63. Lim SW, Hyoung BJ, Piao SG, Doh KC, Chung BH, Yang CW. Chronic cyclosporine nephropathy is characterized by excessive autophagosome formation and decreased autophagic clearance. Transplantation. 2012;94(3):218-225.

64. Ciechomska IA, Gabrusiewicz K, Szczepankiewicz AA, Kaminska B. Endoplasmic reticulum stress triggers autophagy in malignant glioma cells undergoing cyclosporine A-induced cell death. Oncogene. 2013;32(12):1518-1529.

65. Kim HS, Choi SI, Jeung EB, Yoo YM. Cyclosporine A induces apoptotic and autophagic cell death in rat pituitary GH3 cells. PLoS One. 2014;9(10):e108981.

66. Aravindan N, Shaw A. Effect of furosemide infusion on renal hemodynamics and angiogenesis gene expression in acute renal ischemia/reperfusion. Ren Fail. 2006;28(1):25-35.
67. Aravindan N, Aravindan S, Riedel BJ, Weng HR, Shaw AD. Furosemide prevents apoptosis and associated gene expression in a rat model of surgical ischemic acute renal failure. Ren Fail. 2007;29(4):399-407.

68. Fleck C, Heller J. Age-dependent differences in the effect of ischaemia on the rat kidney: prevention of the postischaemic damage by different drugs. Exp Toxicol Pathol. 1993;45(5-6):381-387.

69. Ejaz AA, Mohandas R. Are diuretics harmful in the management of acute kidney injury? Curr Opin Nephrol Hypertens. 2014;23(2):155-160.

70. Esposito C, Grosjean F, Torreggiani M, et al. Sirolimus prevents short-term renal changes induced by ischemiareperfusion injury in rats. Am J Nephrol. 2011;33(3):239-249.

71. Cicora F, Lausada N, Vasquez DN, et al. Protective effect of immunosuppressive treatment before orthotopic kidney autotransplantation. Transpl Immunol. 2011;24(2):107-112.

72. Yang B, Jain S, Pawluczyk IZ, et al. Inflammation and caspase activation in long-term renal ischemia/reperfusion injury and immunosuppression in rats. Kidney Int. 2005;68(5):2050-2067.

73. Fuller TF, Freise CE, Serkova N, Niemann CU, Olson JL, Feng S. Sirolimus delays recovery of rat kidney transplants after ischemia-reperfusion injury. Transplantation. 2003;76(11):1594-1599.

74. Lieberthal W, Fuhro R, Andry CC, et al. Rapamycin impairs recovery from acute renal failure: role of cell-cycle arrest and apoptosis of tubular cells. Am J Physiol Renal Physiol. 2001;281(4):F693-F706.

75. Lui SL, Chan KW, Tsang R, Yung S, Lai KN, Chan TM. Effect of rapamycin on renal ischemia-reperfusion injury in mice. Transpl Int. 2006;19(10):834-839.

76. Ninova D, Covarrubias M, Rea DJ, Park WD, Grande JP, Stegall MD. Acute nephrotoxicity of tacrolimus and sirolimus in renal isografts: differential intragraft expression of transforming growth factor-betal and alpha-smooth muscle actin. Transplantation. 2004;78(3):338-344.

77. Suyani E, Derici UB, Sahin T, et al. Effects of everolimus on cytokines, oxidative stress, and renal histology in ischemiareperfusion injury of the kidney. Ren Fail. 2009;31(8):698-703.

78. Liu M, Agreda P, Crow M, Racusen L, Rabb H. Effects of delayed rapamycin treatment on renal fibrosis and inflammation in experimental ischemia reperfusion injury. Transplant Proc. 2009;41(10):4065-4071.

79. Cicora F, Roberti J, Vasquez D, et al. Preconditioning donor with a combination of tacrolimus and rapamacyn to decrease ischaemia-reperfusion injury in a rat syngenic kidney transplantation model. Clin Exp Immunol. 2012;167(1):169-177.

80. Jolicoeur EM, Qi S, Xu D, Dumont L, Daloze P, Chen H. Combination therapy of mycophenolate mofetil and rapamycin in prevention of chronic renal allograft rejection in the rat. Transplantation. 2003;75(1):54-59.

81. Yang B, Jain S, Ashra SY, Furness PN, Nicholson ML. Apoptosis and caspase-3 in long-term renal ischemia/reperfusion injury in rats and divergent effects of immunosuppressants. Transplantation. 2006;81(10):1442-1450.

82. Sabbatini M, Uccello F, Serio V, et al. Effects of mycophenolate mofetil on acute ischaemia-reperfusion injury in rats and its consequences in the long term. Nephrol Dial Transplant. 2010;25(5):1443-1450.

83. Zhang YX, Zhang JR, Wang ZG. Mycophenolate mofetil affects monocyte Toll-like receptor 4 signaling during mouse renal ischemia/reperfusion injury. Chin Med J (Engl). 2013;126(7): 1224-1229.

84. Yan L, Vatner DE, Kim SJ, et al. Autophagy in chronically ischemic myocardium. Proc Natl Acad Sci U S A. 2005;102(39): 13807-13812. 